\title{
Ocorrência de Neoneura maria (Scudder, 1866) (Odonata: Protoneuridae) para a Província de Santiago de Cuba
}

\author{
Cesar Carriço ${ }^{1,2,4}$, Tatiana Chrysostomo Santos ${ }^{2}$, \\ Janira Martins Costa ${ }^{2}$ \& Adrian David Trapero Quinta ${ }^{3}$ \\ ${ }^{\text {I} P r o g r a m a ~ d e ~ P o ́ s-g r a d u a c ̧ a ̃ o ~ e m ~ B i o l o g i a ~ A n i m a l ~-~ P P G B A, ~ I n s t i t u t o ~ d e ~ B i o l o g i a, ~}$ \\ Universidade Federal Rural do Rio de Janeiro - UFRJ \\ BR 465, Km 7, CEP 23890-000, Seropédica, RJ, Brasil \\ ${ }^{2}$ Departamento de Entomologia, Museu Nacional, Universidade Federal do Rio de Janeiro - UFRJ \\ Quinta da Boa Vista, CEP 20940-040, São Cristóvão, Rio de Janeiro, RJ, Brasil \\ ${ }^{3}$ Departamento de Biología, Facultad de Ciencias Naturales, Universidad de Oriente - UO \\ Alturas de Quintero, Patrício Lumumba, Santiago de Cuba 90500, Cuba \\ ${ }^{4}$ Autor para correspondência: Cesar Carriço,e-mail: carrico82@hotmail.com
}

CARRIÇO, C., SANTOS, T.C., COSTA, J.M. \& QUINTANA, A.D.T. Ocurrence of Neoneura maria (Scudder, 1866) (Odonata: Protoneuridae) for the Province of Santiago de Cuba. Biota Neotrop. 9(4): http://www. biotaneotropica.org.br/v9n4/en/abstract?short-communication+bn01509042009.

\begin{abstract}
During the period 2005-2006 the macroinvertebrates associated with the root system of Eichhornia crassipes (Mart.) Solms, 1883 were studied in the overflow Chalons dam in Santiago de Cuba. The larva of Neoneura maria (Scudder, 1866), endemic for Cuba, reported to the three Sectors of the island, was collected and constitute the first report for the Província de Santiago de Cuba.
\end{abstract}

Keywords: odonata, zygoptera, new report, Cuba, lentic environment.

CARRIÇO, C., SANTOS, T.C., COSTA, J.M. \& QUINTANA, A.D.T. Ocorrência de Neoneura maria (Scudder, 1866) (Odonata: Protoneuridae) para a Província de Santiago de Cuba. Biota Neotrop. 9(4): http://www. biotaneotropica.org.br/v9n4/pt/abstract?short-communication+bn01509042009.

Resumo: Durante o período de 2005-2006 foram estudados os macroinvertebrados associados ao sistema radicular de Eichhornia crassipes (Mart.) Solms, 1883 na represa Chalons de Santiago de Cuba. A larva de Neoneura maria (Scudder, 1866), endêmica de Cuba, registrada para os três Setores da ilha, foi capturada constituindo o primeiro registro para a Província de Santiago de Cuba.

Palavras-chave: odonata, zygoptera, nova ocorrência, Cuba, ambiente lêntico. 


\section{Introdução}

A Ordem Odonata é representada em Cuba, até o presente, por 81 espécies das quais cinco são endêmicas: Neoneura maria (Scudder, 1866), N. carnatica Selys, 1886, Microneura caligata Hagen in Selys, 1886, Protoneura capillaris (Rambur, 1842) e Enallagma truncatum (Gundlach, 1888).

O gênero Neoneura foi criado por Selys (1860), designando como espécie-tipo Neoneura bilinearis. No mesmo trabalho o autor descreveu $N$. rubriventris. Em 1886 Selys publicou cinco novas espécies para o gênero ( $N$. carnatica, $N$. fulvicollis, $N$. rufithorax, $N$. sylvatica e N. waltheri). Calvert (1892-1908) descreveu N. aaroni, N. amelia e $N$. paya para o sudeste do Texas, México e América Central e Scudder (1866) criou Agrion maria, posteriormente transferido para Neoneura por Muttkowski (1910). Williamson (1917) descreveu seis novas espécies ( $N$. denticulata, $N$. esthera, $N$. ethela, $N$. joana, $N$. mariana e $N$. myrthea), redescreveu $N$. maria incluindo ilustrações das espécies já conhecidas e no mesmo ano, Kennedy ilustrou os pênis das espécies de Neoneura. Posteriormente, novas espécies foram descritas isoladamente por Rácenis (1953, 1955), Machado (1975, 1989, 2002, 2003, 2005, 2007), De Marmels (1989), Garrison (1999) e Juillerat (2007). Atualmente o gênero é constituído por 29 espécies (Garrison, 2008), distribuídas desde o sul dos Estados Unidos (Texas) até o norte da Argentina, na América do Sul.

As espécies de Neoneura são pequenas, delicadas, de baixa dispersão e, geralmente, apresentam distribuição restrita. Para Cuba têm-se o registro de apenas duas espécies: $N$. carnatica e $N$. maria, as quais são endêmicas. Estas espécies são morfologicamente semelhantes sendo diferenciadas principalmente pela coloração: $N$. carnatica possui coloração laranja e $N$. maria coloração azul (Alayo, 1968). N. maria foi registrada para o três Setores geográficos de Cuba (Ocidental, Central e Oriental) e N. carnatica para os Setores Ocidental e Central.

Este trabalho tem como objetivo registrar pela primeira vez a ocorrência de N. maria para a Província de Santiago de Cuba. A larva desta espécie ocorre em ambiente lótico, entretanto, o espécime que serviu de base para este estudo foi encontrado em ambiente lêntico, associado às raízes de Eicchornia crassipes (Mart.) Solms, 1883.

\section{Material e Métodos}

As coletas foram realizadas na represa Chalons (Figura 1), localizada ao norte da cidade de Santiago de Cuba, georreferenciada em $75^{\circ} 48^{\prime} 49^{\prime \prime} \mathrm{S}$ e $20^{\circ} 4^{\prime} 12^{\prime \prime} \mathrm{N}$, no período de março de 2005 a março de 2006. O ambiente é tipicamente lêntico, com algumas áreas de leve correnteza. A vegetação aquática constitui-se, principalmente, de E. crassipes (Pontederiaceae) que cobre a maior parte da superfície da água. No local são encontradas, também, as espécies Typha angustifolia (Typhaceae), a Pteridophyta aquática Salvinia auriculata (Salviniaceae) e na zona semiaquática Cyperus rotundus (Cyperaceae). O substrato é lodoso, com muita matéria orgânica e aproximadamente $0,8 \mathrm{~m}$ de profundidade. A localidade apresenta escassa vegetação marginal arbórea e arbustiva com grande incidência luminosa após as 10:00 horas.

Para as coletas dos macroinvertebrados associados a E. crassipes se utilizou a metodologia proposta por Melo et al. (2002) e Meerhoff et al. (2003).

\section{Resultados e Discussão}

Neoneura maria, até o presente, foi encontrada apenas em ambientes lóticos, ocorrendo em rios com mata ciliar e temperaturas entre 22 e $24^{\circ} \mathrm{C}$, nos três Setores Geográficos de Cuba. Suas larvas apresentam hábitos nadadores - trepadores, como a maioria dos

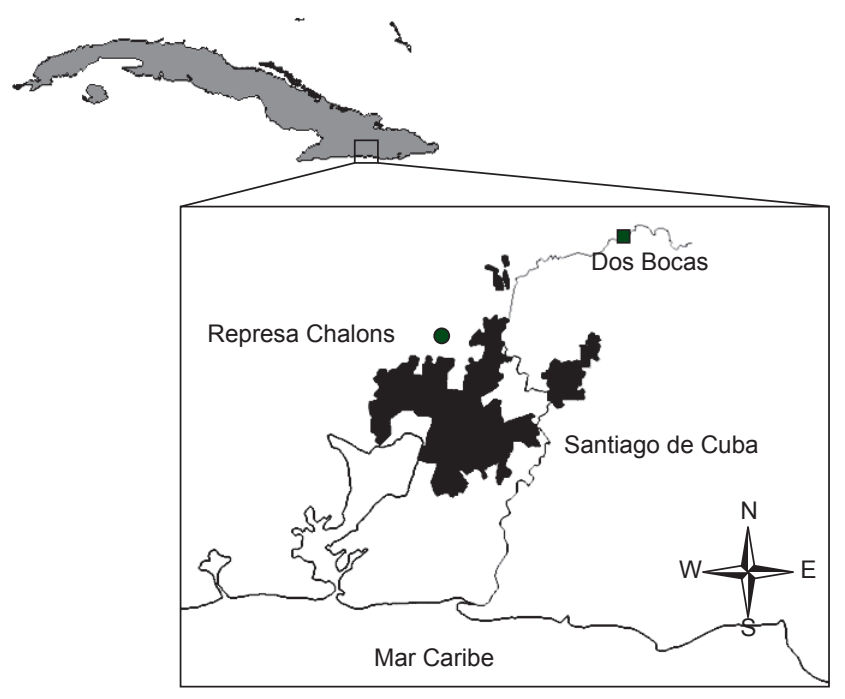

Figura 1. Localização da Represa Chalons, Província de Santiago de Cuba, local de nova ocorrência de Neoneura maria.

Figure 1. Localization of the Chalons Dam, Província de Santiago de Cuba, place of new occurrence of Neoneura maria.

protoneurídeos (Gloyd \& Wright, 1959). Esta espécie está ameaçada de extinção por degradação de seus hábitates naturais (Trapero \& Naranjo, 2003). Na província de Santiago de Cuba um exemplar imaturo foi coletado associado às raízes de E. crassipes, na represa Chalons; o que pode ser justificado através de duas hipóteses: (1) a larva foi carreada até esta localidade e utilizou a macrófita como um substrato de suporte e refúgio ou (2) esta espécie tem preferência por águas correntes, podendo ocasionalmente colonizar ambientes lênticos, sendo tolerante à alterações ambientais. Acreditamos que a segunda hipótese seja mais viável uma vez que exemplares adultos desta espécie foram coletados na mesma localidade, pousados sobre a vegetação aquática. A larva de Neoneura maria caracteriza-se por apresentar o epiprocto ligeiramente visível, paraproctos com 28 a 35 setas na margem ventral, pernas com faixas transversais indistintas e pequeno porte (Trapero \& Naranjo, 2009). O macho adulto de $N$. maria possui cabeça azul pálida, com uma faixa negra transversal ao nível dos ocelos; protórax azul pálido com uma linha superior, uma estria lateral e uma mancha no ápice negra; o sintórax, também azul pálido, possui estrias e máculas negras; abdômen negro iridescente, com máculas azul pálido e extremidade distal azul; asas hialinas, estigma amarelado. A fêmea é semelhante ao macho, porém de coloração mais escura (Alayo, 1968).

\section{Conclusão}

Neoneura maria é registrada pela primeira vez para a província de Santiago de Cuba. Embora esta espécie tenha sido coletada apenas em ambientes lóticos, nos três Setores de Cuba, é provável que esteja colonizando ambientes lênticos, uma vez que na mesma localidade foram capturados larva e adulto.

\section{Agradecimentos}

Ao Dr. Pablo Pessacq da Comisión Nacional de Investigaciones Científicas y Tecnológicas (CONICET), Argentina, pela revisão crítica do manuscrito. À Coordenação de Aperfeiçoamento de Pessoal de Nível Superior (CAPES) e ao Conselho Nacional de Desenvolvimento Científico e Tecnológico (CNPq). À Universidad de Oriente de Cuba. 


\section{Referências Bibliográficas}

ALAYO, P. 1968. Las Libélulas de Cuba (Insecta - Odonata). Torreia 2(1): 1-102.

CALVERT, P.P. 1892-1908. Odonata, Neuroptera (F.D. Godman, ed.). In Biol. Centr. Amer., London, p. 17-420.

DE MARMELS, J. 1989. Odonata or dragonflies from Cerro de la Neblina. B. Acad. Cienc. Fis., Matemat. Natur. 25(1): 1-78.

GARRISON, R.W. 1999. The genus Neoneura, with key and description of a new species, Neoneura jurzitzai Spec. Nov. (Zygoptera: Protoneuridae). Odonatologica 28(4): 343-375.

GARRISON, R.W. 2008. A synonymic list of the new world Odonata. http:// www.odonatacentral.org/ (último acesso em 16/07/2009).

GLOYD, L.K. \& WRIGHT, M. 1959. Odonata. John Wiley \& Sons, Inc. Fresh-Water Biology 34(1): 915-940.

JUILLERAT, L. 2007. Neoneura angelensis sp. nov. from French Guyana (Odonata: Protoneuridae). Int. J. Odonatol. 10(2): 203-208.

KENNEDY, C.H. 1917. Notes on the penes of Zygoptera (Odonata). Entomol. News 28(3): 289-294.

MACHADO, A.B.M. 1975. Neoneura schreiberi nova espécie da região amazônica (Odonata - Protoneuridae). Cienc. Cultura 27(7): 764-766.

MACHADO, A.B.M. 1989. Studies on neotropical Protoneuridae. 8. Neoneura desana spec. nov. from the amazonian region (Odonata, Zygoptera). Rev. Bras. Entomol. 33(2): 225-228.

MACHADO, A.B.M. 2002. Neoneura lucas spec. nov. from brazilian Pantanal (Zygoptera: Protoneuridae). Odonatologica 31: 199-204.

MACHADO, A.B.M. 2003. Studies on neotropical Protoneuridae.13. Neoneura moorei spec. nov. from the Amazonian region of Brazil. Odonatologica 32: 125-130.

MACHADO, A.B.M. 2005. Studies on neotropical Protoneuridae. 19. Two new species of Neoneura from Southern Brazil (Odonata, Protoneuridae). Iheringia Ser. Zool. 95(4): 405-409.

MACHADO, A.B.M. 2007. Studies on Neotropical Protoneuridae. Neoneura kiautai spec. nov. from southeastern Brazil (Zygoptera, Protoneuridae)
(B.K. Tyagi, ed.). In: Odonata: biology of dragonflies. Scientific Publisher, Jodhpur, p. 25-32.

MEERHOFF, M., MAZZEO, N., MOSS, B. \& RODRÍGUEZ, L. 2003. The structuring role of free-floating versus submerged plants in a subtropical shallow lake. Aquat. Ecol. 37 (1): 377-391.

MELO, S., TAKEDA, A. \& ONKOLSKI, A. 2002. Seasonal dynamics of Callibaetis willineri (Ephemeroptera, Baetide) associated with Eichhornia azurea (Pontederiaceae) in Guaraná lake of the upper Paraná river, Brasil. Hydrobiologia 470(15): 57-62.

MUTTKOWSKI, R.A. 1910. Catalogue of the Odonata of North America. B. Public. Mus. Milwaukee 1(1):1-207.

RÁCENIS, J. 1953. Una nueva espécie del genero Neoneura (Odonata:Protoneuridae) de Venezuela. Acta Biol. Venezol. 1(9): 155-158.

RÁCENIS, J. 1955. Los representantes Venezolanos de la família Protoneuridae (Odonata). Mem. Soc. Cienc. Natur. La Salle 15: 48-62.

SCUDDER, S.H. 1866. Notes upon some Odonata from the Isle of Pines. P. Boston Soc. Nat. Hist. 10(1):187-198.

SELYS-LONGCHAMPS, E. 1860. Synopsis de Agrionines, dernière légion: Protonevra. B. Cl. Sci. Ac. Roy. Belg. (II) 10(9-10):431-462.

SELYS-LONGCHAMPS, E. 1886. Revision du Synopsis des Agrionines. B. Cl. Sci. Ac. Roy. Belg. 1(4):1-233.

TRAPERO, A. \& NARANJO, C. 2003. Revision of the order Odonata in Cuba. B. Amer. Odonatol. 2(7): 23- 40.

TRAPERO, A. \& NARANJO, C. 2009. Clave para la identificación de especies de Odonata en estado larval de Cuba. Boletín Sociedad Entomológica Aragonesa. 44(1): 459-467.

WILLIAMSON, E.B. 1917. The genus Neoneura (Odonata). T. Am. Entomol. Soc. 43 (1): 211-246. 